\title{
Imbalance and Falls in Elderly: Review of Literature
}

\author{
Yogendra Singh Chauhan
}

\begin{abstract}
The purpose of review is to emphasize on the causes and assessment of elderly with imbalance and fall. Rising elderly population in future leading to increase in falls episode and associated injury will bring neurologists, otolaryngologists, cardiologists, psychiatrists, orthopedicians and occupational therapists under one umbrella for the management of elderly with balance disorder. This will increase in interspeciality mutualism and understanding ultimately leading to achieve goal to help our elderly to have better life.
\end{abstract}

Keywords: Imbalance and fall, Elderly, Otolaryngologists.

How to cite this article: Chauhan YS. Imbalance and Falls in Elderly: Review of Literature. Int J Otorhinolaryngol Clin 2013;5(3):139-141.

\section{Source of support: Nil}

Conflict of interest: None declared

\section{INTRODUCTION}

With the normal aging process, humans experience changes in the sensory systems, the central and peripheral nervous systems and in muscles and joints. These multisystem losses affects the functions necessary for balance. In the future, an increasing number of elderly individuals with age related dizziness and loss of balance will be referred to otolaryngologists, although most training programs teach the management of vestibular disorders, frequently balance loss because of presbystasis is not thoroughly addressed. To properly evaluate and manage these patients, otolaryngologist must go beyond the usual head-neck examination and evaluate all the sensory systems that contribute to balance, including vision, vestibular, proprioceptive senses, and the patient's ability to process this information and produce effective balance and locomotion (Fig. 1). Falls and the resulting injuries have become one of the most serious health issues for the elderly. A definite pattern is seen to falls in the elderly as 'post falls syndrome'. ${ }^{1}$ Post fall syndrome is defined as 'loss of confidence and voluntary restrictions on activity that is dramatically out of proportion to physical injuries sustained. The number of falls and severity of injuries have been shown to increase with age and limited movements.

\section{DISCUSSION}

More than half of all elderly falls occur in the home. The most common site of falls is bedroom mainly getting into or out of bed, which accounts for approximately one-third

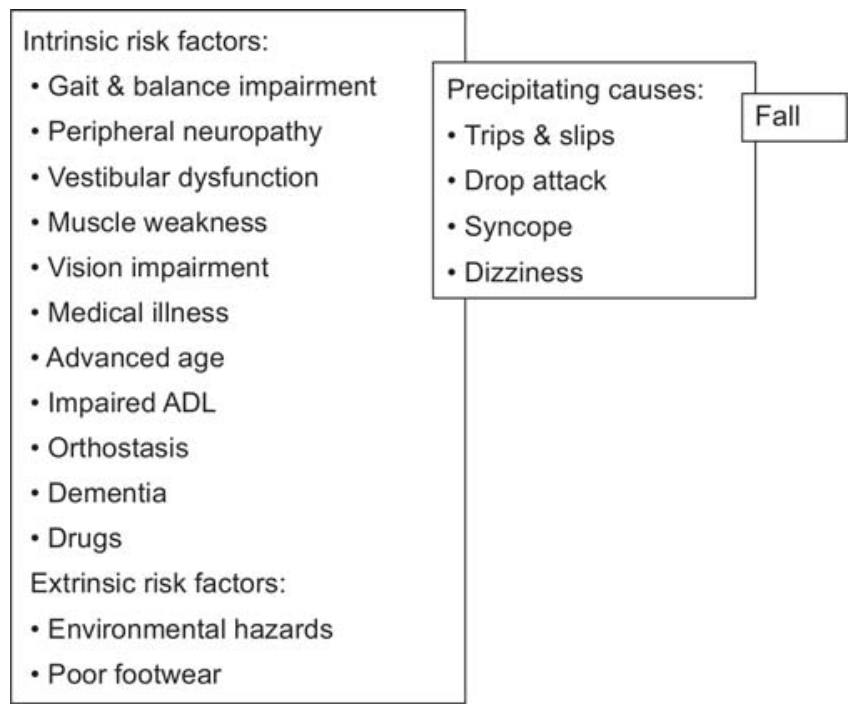

Fig. 1: The multifactorial and interacting causes of fall

of these home falls. Other home sites include change in surface or lighting (12\%); bathroom (10\%); kitchen (10\%); living room (8\%); utility room (5\%). ${ }^{2}$

Changes associated with aging in vestibular system are reduction in the sensitivity of the semicircular canals, a decrease in the number of hair cells in the sensory epithelium, decline in number of first degree order neuron to vestibular nucleus.

Initially, central compensation occurs by reduction in the inhibitory system mediated by cerebellum. ${ }^{3}$ Long term, however, the range of response is greatly reduced. The result is that elderly respond fairly well to slower ranges of head movement, gait and posture, but more rapid movements cannot be satisfactorily. Decreased flexibility and strength of muscles and bones further reduce the ability to respond. The outcome is frequently a fall.

Often, elderly have poor nutrition affecting overall well being. Studies have shown elderly with greater degree of social and physical activities present a lowered risk of fall. ${ }^{4}$ Race and gender may also play a role; women fall more than men, whites fall more frequently than nonwhites and person older than 65 , fall rate increases proportional to age.

Vascular diseases represent most common nonvestibular cause of dizziness and balance loss in the elderly. Vertebrobasilar artery and carotid artery stenosis secondary to atherosclerosis can lead to transient ischemic attack (TIAs) and stroke (CVAs). Coronary artery disease (CADs) 
can lead to arrhythmias, myocardial infarction, etc. Gait and balance disorders also are common among older adults, and affect between 20 and 50\% of people who are older than 65 years $5,6,16,17$ Among nursing home populations, nearly three quarters of residents require assistance with ambulation or are completely unable to ambulate. ${ }^{7,18}$ Gait and balance impairments were a significant risk factor for falls, and were associated with about a threefold increased risk for falling; the use of an assistive device for ambulation was associated with a 2.6-fold increased risk for falling.

Head traumas, epilepsy, multiple sclerosis, cerebellar ataxia are some neurological disorders leading to loss of balance function. Diabetes mellitus leading to peripheral neuropathy and atherosclerosis can be responsible for disequilibrium and balance disorder. Hypertension and hypotension, reduced visual acuity, arthritis are also related to balance dysfunction. Visual impairment increases the risk for falling about 2.5 times. At least $18 \%$ of noninstitutionalized persons who are 70 years and older have substantial visual impairment. ${ }^{8,19}$ The primary causes include cataracts, glaucoma, and macular degeneration. Arthritis, the most common chronic condition in persons 70 years and older in the United States, ${ }^{8,19}$ increases the risk for falling about 2.4 times. The relationship between arthritis and falls most likely is related to the gait impairment and weakness that often are associated with arthritis. Functional impairment, usually indicated by the inability to perform basic activities of daily living (ADLs; e.g. dressing, bathing, eating), doubles the risk for falling. In the community, ADL impairment affects $20 \%$ of persons who are older than age 70 s. ${ }^{8,19}$ In the nursing home setting, the prevalence of functional impairment is much higher; $96 \%$ of nursing home residents require assistance with bathing and 45\% require assistance with eating. ${ }^{7,18}$

Psychiatric disorders like anxiety-depression disorder, panic disorder, agoraphobia should be evaluated in elderly in parallel to medical condition as remain hidden as the primary cause and also may lead to failure of balance retraining therapy. Depression is associated with about a two-fold increased risk for falling. Although the relationship between depression and falls is not well studied, depression may result in inattention to the environment, or cause more risk-taking behaviors. Conversely, it may be a reaction to previous fall related morbidity and not be an actual causative risk at all. Additionally, psychotropic medications increase fall risk. ${ }^{9,10,20,21}$ Common risk factors have been identified for depression and falls (i.e. poor self-rated health, cognitive impairment, functional impairment, slow gait speed). ${ }^{11,22}$ Mild depressive symptoms occur in close to one quarter of the older population, and about $5 \%$ of this population suffers major depression. ${ }^{12}$ Cognitive impairment almost doubles the risk for falling. In a recent study among residents of 59 nursing homes, the unadjusted fall rate for residents who had dementia was 4.05 falls per year compared with 2.33 falls per year for residents who did not have dementia (adjusted relative risk, 1.74). ${ }^{13}$ Confusion and cognitive impairment are cited frequently as causes of falls, and may reflect an underlying systemic or metabolic process (e.g. electrolyte imbalance, fever), as well as a dementing illness. Dementia can increase falls by impairing judgment, visuospatial perception, and orientation ability. Falls also occur when demented residents wander, attempt to get out of wheelchairs, or climb over bedside rails. Cognitive impairment affects between 5 and 15\% of persons who are older than age 65, and the prevalence increases with age and among institutionalized population.

Medications like hypoglycemic agents, psychotropic drugs, analgesics, antiepileptics and narcotics can lead to balance symptoms. Vestibular suppressive medications cause reduction of already lessened vestibular function and often making symptoms worse. In addition they also reduce ability of the brain to compensate vestibular injury.

Elderly with presbystasis unlike younger patient with balance disorder complain of imbalance that is gradually progressive, an unsteady walk, disequilibrium, and frequent light-headedness or presyncopal sensations without loss of consciousness. They complain these symptoms when standing or walking and improvement when sitting or lying down.

Computer dynamic posturography ( CDP ) is excellent testing modality for determining age-related balance loss and predicting falls risk. ${ }^{14,15}$ If CDP is not available, other test include the Romberg test, CTSIB (clinical test of sensory integration in balance), ${ }^{16}$ MFES (modified falls efficacy scale). ${ }^{17}$ Chair rising performance is quick screening tool in which person is asked to rise out of an armless chair without using hand. If task require more than 2 seconds for one trial or more than 18.5 seconds for five successive trials, there is increased chance of falling. ${ }^{18}$ ENG is also important tool for evaluating vestibular sensitivity. ${ }^{19}$ Vestibular rehabilitation therapy has shown to highly effective treatment for elderly with presbyastasis. ${ }^{20}$

\section{SUMMARY}

Studies have shown that it is possible to identify persons who are at a substantially increased risk for sustaining a fall or fall-related injury by detecting the presence of risk factors. Many, if not all, of these risk factors are amenable to treatment or rehabilitative approaches to ameliorate them. Consequently, risk factor identification seems to be a 
promising first step in developing effective fall-prevention programs that are targeted to high-risk patients. To assist clinicians in the assessment of fall risk, evidence-based clinical practice guidelines on fall prevention and treatment were published by the American and British Geriatrics Societies. ${ }^{21}$ Among other things, the guidelines recommend that a fall risk assessment be an integral part of primary healthcare for older persons, with the intensity of the assessment tailored to the target population (e.g. low-risk vs high-risk individuals). Several published fall risk assessment tools are available for quantifying fall risk for older persons at home and in institutional settings. An analytic review of these assessment tools recommended several that seem to be valid and potentially useful. ${ }^{22}$ Although the importance of fall risk factor identification is accepted generally, the question of how best to modify these risk factors to prevent falls continues to be a challenge for clinicians and researchers.

\section{REFERENCES}

1. Murphy J, Isaacs B. The post fall syndrome. A study of 36 elderly patients. Gerontology 1982;28:265.

2. Tideiksaar R. Falling in old age: its prevention and management, 2nd ed. Vol 22 of springer series on adulthood and aging, Issue 22 of Adulthood and Aging Series, New York, Springer Publishing Co 1997.

3. Nakayama M, Helfert RH, Konrad HR. Scanning electron microscopic evaluation of age-related changes in vestibular epithelium. Otolaryngol Head Neck Surg 1995;111:799.

4. Girardi M, Konrad HR, Amin M, Hughes LF. Predicting fall risks in an elderly population: computer dynamic posturography versus electronystagmography test results. Laryngoscope 2001;111(9):1528-1532.

5. Alexander NB. Gait disorders in older adults. J Am Geriatr Soc 1996;44(4):434-451.

6. Sudarsky L. Geriatrics: gait disorders in the elderly. N Engl J Med 1990;322:1441-1446.

7. Sahyoun NR, Pratt LA, Lentzner H, Dey A, Robinson KN. The Changing Profile of Nursing Home Residents: 1985-1997. Aging Trends; No.4. Hyattsville, Maryland: National Center for Health Statistics. 2001
8. Kramarow E, Lentzner H, Rooks R, Weeks J, Saydah S. Health and Aging Chartbook. Health, United States, 1999. Hyattsville, Maryland: National Center for Health Statistics. 1999.

9. Leipzig RM, Cumming RG, Tinetti ME. Drugs and falls in older people: a systematic review and meta-analysis: I. Psychotropic drugs. J Am Geriatr Soc 1999;47:30-39.

10. Leipzig RM, Cumming RG, Tinetti ME. Drugs and falls in older people: a systematic review and meta-analysis: II. Cardiac and analgesic drugs. J Am Geriatr Soc 1999;47:40-50.

11. Biderman A, Cwikel J, Fried AV, et al. Depression and falls among community dwelling elderly people: a search for common risk factors. J Epidemiol Community Health 2002;56:631-636.

12. Blazer D, Williams CD. Epidemiology of dysphoric and depression in an elderly population. Am J Psychiatry 1980;137:413-417.

13. Van Doorn C, Gruber-Baldini AL, Zimmerman S, et al. Dementia as a risk factor for falls and fall injuries among nursing home residents. J Am Geriatr Soc 2003;51:1213-1218.

14. Aging trends. Hyattsville (MD): National Center for Health Statistics; 2001;(4).

15. Baloh RW, Spain S, Socotch TM. Posturography and balance problems in older people. J Am Geriatr Soc 1995;43:638.

16. Skinner HB, Barrach RL, Cook SD. Age-related declines in proprioception. Clin Orthop 1984;184:208.

17. Horak FB. Clinical measure of postural control in adults. Phys Ther 1987;67:1881.

18. Hill KD, Schwarz JA, Kalogeropoulos AJ. Fear of falling revisited. Arch Phys Med Rehabil 1996;77:1025.

19. Merrill SS, Seeman TE, Kasl SV. Gender differences in the comparison of self-reported disability and performance measures. J Gerontol 1997Jan;52(1):M19-26.

20. McPherson DL, Whitacker SL. Disequilibrium of aging; practical management of dizzy patients, Lippincott Williams \& Wilkins, Philadelphia, 2001. p. 269-298.

21. American Geriatrics Society, British Geriatrics Society, American Academy of Orthopaedic Surgeons Panel on Falls Prevention. Guideline for the prevention of falls in older persons. J Am Geriatr Soc 2001;49:664-672.

22. Perell KL, Nelson A, Goldman RL, et al. Fall risk assessment measures: an analytic review. J Gerontol 2001;56A:M761-766.

\section{ABOUT THE AUTHOR}

\section{Yogendra Singh Chauhan}

Assistant Professor, Department of ENT, Rama Medical College Kanpur, Uttar Pradesh, India, e-mail: dr.yogendrared@yahoo.com 